\section{Re: Trimethoprim-sulfamethoxazole or Clindamycin for Community-Associated MRSA (CA-MRSA) Skin Infections}

To the Editor: I read the article by Frei et $\mathrm{al}^{1}$ with great interest and would like to emphasize the important role of incision and drainage (I\&D) for management of skin and soft tissue infection (SSTI) caused by communityassociated methicillin-resistant Staphylococcus aureus (CAMRSA). As a general rule in infectious diseases, any antibiotics, no matter how strong and broad-spectrum they are, will not work effectively unless the infected source is properly drained.

Since the emergence of CA-MRSA as the important cause of infections in both outpatient and inpatient settings, there is growing evidence that supports the use of non- $\beta$-lactam antibiotics, including trimethoprim-sulfamethoxazole, clindamycin, and tetracyclines for treatment of CA-MRSA SSTIs. ${ }^{2-5}$ Among patients with drainable abscesses caused by CA-MRSA, treatment options can be either I\&D or a combination of I\&D and antibiotics. However, there have been no clinical studies to compare the efficacy between these treatment options. $^{2-5}$ The conclusion by Frei et $\mathrm{al}^{1}$ that combination of I\&D and antibiotics is more effective than I\&D alone may not be totally accurate because of the following reasons. First, it is premature to draw this conclusion from this study given its limitations, including its retrospective nature, the small sample size, and that the subgroup analysis of patients undergoing I\&D was not predefined. Secondly, characteristics between the groups receiving combination of $I \& D$ and antibiotics and I\&D alone were not compared to prove similarities. Third, disease severity, which is one of the most important confounders, was not adjusted. Patients with mild infection could be treated with I\&D alone without the need for antibiotics, whereas the role of antibiotics would be obvious in more severe cases. Unfortunately, there are no common criteria or scale of severity for SSTIs. Thus, it is difficult to compare results between studies based on patient severity. Some of the characteristics that can affect outcomes and should be included in the criteria or severity scale are the size of the lesion, the number of lesions, and presence of concurrent ulcer or abscess, fever, and sepsis. ${ }^{2}$ Lastly, a study previously published by myself and my colleague demonstrated a high treatment success rate $(85 \%)$ using cephalexin among patients with skin abscesses who were undergoing I\&D despite that
$70 \%$ of the cultures grew CA-MRSA, which were resistant to cephalexin. ${ }^{2}$ This finding is consistent with the findings from the study by Rajendran et al, ${ }^{6}$ which with the authors contrasted their data.

Given the potential adverse reactions and resistance created by the overuse of antibiotics, I encourage proper selection of patient populations that would benefit from antibiotic treatment of CA-MRSA SSTIs. A standardized disease severity scale for SSTIs and prospective randomized studies are needed to assess and compare the efficacy between I\&D alone and in combination with antibiotics for patients with CA-MRSA SSTIs.

Thana Khawcharoenporn, MD

Section of Infectious Diseases

Rush University Medical Center

Chicago, IL thanak30@yahoo.com

\section{References}

1. Frei CR, Miller ML, Lewis JS II, et al. Trimethoprimsulfamethoxazole or clindamycin for community-associated MRSA (CA-MRSA) skin infections. J Am Board Fam Med 2010;23:714-9.

2. Khawcharoenporn T, Tice A. Empiric outpatient therapy with trimethoprim-sulfamethoxazole, cephalexin, or clindamycin for cellulitis. Am J Med 2010;123:942-50.

3. Ruhe JJ, Smith N, Bradsher RW, Menon A. Communityonset methicillin-resistant Staphylococcus aureus skin and soft-tissue infections: impact of antimicrobial therapy on outcome. Clin Infect Dis 2007;44:777-84.

4. Ruhe JJ, Menon A. Tetracyclines as an oral treatment option for patients with community onset skin and soft tissue infections caused by methicillin-resistant Staphylococcus aureus. Antimicrob Agents Chemother 2007;51:3298-303.

5. Szumowski JD, Cohen DE, Kanaya F, Mayer KH. Treatment and outcomes of infections by methicillin-resistant Staphylococcus aureus at an ambulatory clinic. Antimicrob Agents Chemother 2007;51:423-8.

6. Rajendran PM, Young D, Maurer T, et al. Randomized, double-blind, placebo-controlled trial of cephalexin for treatment of uncomplicated skin abscesses in a population at risk for community-acquired methicillin-resistant Staphylococcus aureus infection. Antimicrob Agents Chemother 2007; $51: 4044-8$

doi: 10.3122/jabfm.2011.03.100305

The above letter was referred to the author of the article in question, who offers the following reply. 